\title{
On a mechanism realizing quark mass hierarchy
}

\author{
Yoshiharu KAWAMURA* \\ Department of Physics, Shinshu University, \\ Matsumoto 390-8621, Japan
}

April 27, 2020

\begin{abstract}
We reconsider a generation of up-type quark mass hierarchy in the standard model and clarify how a mechanism works to realize the hierarchy without severe fine tuning.
\end{abstract}

\section{Introduction}

It is expected that the fermion mass hierarchies in the standard model (SM) are elegantly understood from unknown features, e.g., flavor symmetries and a structure behind texture zeros, based on the top-down approach [1, 2, 3, 4, 5, 6]. The flavor structure of quarks and leptons has been studied intensively, using various flavor symmetries [6, 7, 8, 9, 10, 11, 12, 13, 14. It is important not only to identify flavor symmetries but also to figure out what's behind their breaking, because no exact flavor-dependent symmetries exist in the SM [15, 16]. The bottom-up approach has also been used [17, 18, 19, 20, 21, 22, 23].

It is mostly believed that the fermion mass hierarchies are derived without severe fine tuning. In other words, tiny Yukawa couplings should not appear as a result of fine tuning among parameters of $O(1)$ size. Hence, it is conjectured that there exist tiny parameters at a more fundamental level, and an excellent mechanism works to generate a hierarchical structure on physical parameters.

In this letter, we reconsider a generation of up-type quark mass hierarchy in the SM and clarify how a mechanism works to realize the hierarchy without severe fine tuning, based on the following ideas. The SM is an effective quantum field theory (QFT), and QFT is a tool or a framework to describe quantum phenomena in an efficient manner. Parameters in the SM Lagrangian are not necessarily fundamental but effective ones, and a tininess of some physical parameters is naturally understood by using more fundamental parameters.

The outline of this letter is as follows. In the next section, we present our basic idea in general terms. We examine up-type quark mass hierarchy in Sect. 3. In the last section, we give conclusions and discussions.

*E-mail: haru@azusa.shinshu-u.ac.jp 


\section{Basic idea}

We show that a hierarchy among physical parameters in magnitude can be generated at a tree level without severe fine tuning, when more fundamental parameters exist. We study an effective QFT described by the Lagrangian density:

$$
\mathscr{L}_{\mathrm{QFT}}=\sum_{\alpha} \frac{c_{\alpha}}{\Lambda^{d_{\alpha}-4}} O_{\alpha}
$$

where $c_{\alpha}$ are dimensionless parameters, $\Lambda$ is a cutoff scale, and $O_{\alpha}$ are operators with mass dimensions $d_{\alpha}$. We assume that particles are weakly coupled, i.e., $\left|c_{\alpha}\right| \leq O(1)$.

Let $c_{\alpha}$ be classified into two categories. One is a set of parameters that are physical in itself, e.g., gauge couplings in the SM. The other is a set of parameters where physical parameters $\tilde{C}_{a}$ originate from, after redundant ones (unphysical ones) are eliminated, e.g., Yukawa coupling matrices $y_{i j}^{(f)}$ in the SM. Hereafter, we focus on a second one. When there appears a hierarchy among $\tilde{C}_{a}$ in magnitude or $\tilde{C}_{a}$ contain a tiny parameter such as up quark Yukawa coupling $y_{u}$, a cancellation among $c_{\alpha}$ is, in general, needed to derive a tiny one with $\left|\tilde{C}_{a}\right| \ll O(1)$ from $\left|c_{\alpha}\right|=O(1)$. In the following, we analyze this feature in a quantitative way.

First, we regard $c_{\alpha}$ as fundamental parameters, and introduce a measure of fine tuning defined by

$$
\Delta_{c_{\alpha}} \tilde{C}_{a} \equiv\left|\frac{\partial \ln \tilde{C}_{a}}{\partial \ln c_{\alpha}}\right|=\left|\frac{\partial \tilde{C}_{a}}{\partial c_{\alpha}} \frac{c_{\alpha}}{\tilde{C}_{a}}\right|
$$

Here and hereafter no summations on $\alpha$ and $a$ are done. The value of $\Delta_{c_{\alpha}} \tilde{C}_{a}$ implies a necessary cancellation of first part of that. Or the smaller $\Delta_{c_{\alpha}} \tilde{C}_{a}$ are, the less a degree of fine tuning is. For instance, in a case with $\left|\partial \tilde{C}_{a} / \partial c_{\alpha}\right|=O(1)$ and $\left|c_{\alpha}\right| \gg\left|\tilde{C}_{a}\right|$ for some $\tilde{C}_{a}$, we have $\Delta_{c_{\alpha}} \tilde{C}_{a} \gg O(1)$ and need severe fine tuning among $c_{\alpha}$ to obtain $\tilde{C}_{a}{ }^{2}$

We give a simple example that $\tilde{C}_{a}$ are given as linear combinations of $c_{\alpha}$ such that $\tilde{C}_{1}=\sum_{\alpha=1}^{3} A_{1 \alpha} c_{\alpha}$ and $\tilde{C}_{2}=\sum_{\alpha=1}^{3} A_{2 \alpha} c_{\alpha}$. We assume that $A_{1 \alpha}$ and $A_{2 \alpha}$ are irrelevant to $c_{\alpha}$, and their magnitudes are given by $\left|A_{1 \alpha}\right|=O(1),\left|A_{2 \alpha}\right|=O(1)$, and $\left|c_{\alpha}\right|=O(1)$. In the presence of $\left|\tilde{C}_{1}\right|=O(1) \gg\left|\tilde{C}_{2}\right|$, degrees of fine tuning are estimated as

$$
\Delta_{c_{\alpha}} \tilde{C}_{1}=\left|A_{1 \alpha} \frac{c_{\alpha}}{\tilde{C}_{1}}\right|=O(1), \Delta_{c_{\alpha}} \tilde{C}_{2}=\left|A_{2 \alpha} \frac{c_{\alpha}}{\tilde{C}_{2}}\right| \gg O(1) .
$$

The second relation means that severe fine tuning is needed to derive $\tilde{C}_{2}$.

Next, we consider a case with more fundamental parameters. They are classified into two categories, $\left\{f_{k}\right\}$ and $\left\{\varepsilon_{l}\right\}$, based on their magnitudes, i.e., $f_{k}=O(1)$ and $\varepsilon_{l} \ll O(1)$. Here, their values are taken as positive ones. For example, $f_{k}$ emerge as vacuum expectation values (VEVs) of some scalar fields such as moduli fields $\phi_{k}^{(\mathrm{M})}$, i.e., $f_{k}=\left|\left\langle\phi_{k}^{(\mathrm{M})}\right\rangle\right| / M$.

\footnotetext{
${ }^{1}$ This type of measure is originally proposed to quantify the degree of fine tuning on the Higgs boson mass among soft supersymmetry breaking parameters [24].

${ }^{2}$ The dependency of $\tilde{C}_{a}$ in $c_{\alpha}$ can be restricted by imposing on a condition such as $\Delta_{c_{\alpha}} \tilde{C}_{a} \leq O(1)$. The finite version is used in a bottom-up approach based on 'stability' principle [19] 20]. Here, the stability principle means that a tiny parameter should not be sensitive to a change of fundamental parameters.
} 
Here, $M$ is a fundamental scale such as a string scale or the Planck scale. Then, the magnitudes of $f_{k}$ are $O(1)$, if those of $\left\langle\phi_{k}^{(\mathrm{M})}\right\rangle$ are $O(M) . \varepsilon_{l}$ are defined by $\varepsilon_{l} \equiv\left|\left\langle\varphi_{l}\right\rangle\right| / M$, and their magnitudes are much less than $O(1)$, when $\left|\left\langle\varphi_{l}\right\rangle\right| \ll M$.

For simplicity, we examine a case with two fundamental parameters $f$ and $\varepsilon$ such that $f=O(1) \gg \varepsilon$. In this case, $\tilde{C}_{a}$ are functions of $f$ and $\varepsilon$, i.e., $\tilde{C}_{a}=\tilde{C}_{a}(f, \varepsilon)$, and degrees of fine tuning are measured by

$$
\Delta_{f} \tilde{C}_{a} \equiv\left|\frac{\partial \ln \tilde{C}_{a}}{\partial \ln f}\right|=\left|\frac{\partial \tilde{C}_{a}}{\partial f} \frac{f}{\tilde{C}_{a}}\right|, \quad \Delta_{\varepsilon} \tilde{C}_{a} \equiv\left|\frac{\partial \ln \tilde{C}_{a}}{\partial \ln \varepsilon}\right|=\left|\frac{\partial \tilde{C}_{a}}{\partial \varepsilon} \frac{\varepsilon}{\tilde{C}_{a}}\right| .
$$

If the following conditions fulfill,

$$
\left|\frac{\partial \tilde{C}_{a}}{\partial f}\right| \leq O\left(\left|\tilde{C}_{a}\right|\right), \quad\left|\frac{\partial \tilde{C}_{a}}{\partial \varepsilon}\right| \leq O\left(\frac{\left|\tilde{C}_{a}\right|}{\varepsilon}\right)
$$

$\Delta_{f} \tilde{C}_{a} \leq O(1)$ and $\Delta_{\varepsilon} \tilde{C}_{a} \leq O(1)$ are derived, and then tiny parameters can be obtained without severe fine tuning. This feature is understood from a viewpoint of perturbation, i.e., effects of $\varepsilon$ can be perturbatively incorporated, as follows. For $\left|\tilde{C}_{a}\right|=O(1), \tilde{C}_{a}$ are, in general, expanded as power series of $\varepsilon$ :

$$
\tilde{C}_{a}=\sum_{n=0}^{\infty} \tilde{C}_{a, n}(f) \varepsilon^{n}
$$

where $\left|\tilde{C}_{a, 0}\right|=O(1)$. If $\left|\partial \tilde{C}_{a, 0} / \partial f\right| \leq O(1)$ and $\left|\tilde{C}_{a, 1}\right| \leq O(1)$ hold, $\Delta_{f} \tilde{C}_{a} \leq O(1)$ and $\Delta_{\varepsilon} \tilde{C}_{a} \leq$ $O(1)$ are derived. For $\left|\tilde{C}_{a}\right|=O(\varepsilon) \ll O(1), \tilde{C}_{a}$ are expanded as power series of $\varepsilon$ :

$$
\tilde{C}_{a}=\sum_{n=1}^{\infty} \tilde{C}_{a, n}(f) \varepsilon^{n}
$$

where $\left|\tilde{C}_{a, 1}\right|=O(1)$. In this case, $\left|\partial \tilde{C}_{a} / \partial \varepsilon\right|=O(1)$ holds. If $\left|\partial \tilde{C}_{a, 1} / \partial f\right| \leq O(1)$ holds, $\Delta_{f} \tilde{C}_{a} \leq O(1)$ and $\Delta_{\varepsilon} \tilde{C}_{a}=O(1)$ are derived. Note that the term with $n=0$ is missing in Eq. (7), and its absence could be due to the existence of some symmetries.

Let us explain the above feature by way of $c_{\alpha}=c_{\alpha}(f, \varepsilon)$. We assume that $c_{\alpha}(f, \varepsilon)$ originate from independent terms in a more fundamental theory, and $c_{\alpha}$ are written by

$$
c_{\alpha}=c_{\alpha}^{(1)}(f)+c_{\alpha}^{(\varepsilon)}(f, \varepsilon),
$$

where $\left|c_{\alpha}^{(1)}\right|=O(1),\left|c_{\alpha}^{(\varepsilon)}\right|=O(\varepsilon),\left|\partial c_{\alpha}^{(1)} / \partial f\right|=O(1),\left|\partial c_{\alpha}^{(\varepsilon)} / \partial f\right|=O(\varepsilon)$ and $\left|\partial c_{\alpha}^{(\varepsilon)} / \partial \varepsilon\right|=O(1)$. Then, $\Delta_{f} \tilde{C}_{a}$ and $\Delta_{\varepsilon} \tilde{C}_{a}$ are calculated as

$$
\Delta_{f} \tilde{C}_{a}=\left|\sum_{\alpha}\left(\frac{\partial \tilde{C}_{a}}{\partial c_{\alpha}^{(1)}} \frac{\partial c_{\alpha}^{(1)}}{\partial f} \frac{f}{\tilde{C}_{a}}+\frac{\partial \tilde{C}_{a}}{\partial c_{\alpha}^{(\varepsilon)}} \frac{\partial c_{\alpha}^{(\varepsilon)}}{\partial f} \frac{f}{\tilde{C}_{a}}\right)\right|, \Delta_{\varepsilon} \tilde{C}_{a}=\left|\sum_{\alpha} \frac{\partial \tilde{C}_{a}}{\partial c_{\alpha}^{(\varepsilon)}} \frac{\partial c_{\alpha}^{(\varepsilon)}}{\partial \varepsilon} \frac{\varepsilon}{\tilde{C}_{a}}\right|,
$$

respectively. If the following conditions fulfill,

$$
\left|\sum_{\alpha} \frac{\partial \tilde{C}_{a}}{\partial c_{\alpha}^{(1)}} \frac{\partial c_{\alpha}^{(1)}}{\partial f}\right| \leq O\left(\left|\tilde{C}_{a}\right|\right), \quad\left|\frac{\partial \tilde{C}_{a}}{\partial c_{\alpha}^{(\varepsilon)}}\right| \leq O\left(\frac{\left|\tilde{C}_{a}\right|}{\varepsilon}\right)
$$


we find that $\Delta_{f} \tilde{C}_{a} \leq O(1)$ and $\Delta_{\varepsilon} \tilde{C}_{a} \leq O(1)$. The point is that $\tilde{C}_{a}$ are not functions of $c_{\alpha}$ but $c_{\alpha}^{(1)}$ and $c_{\alpha}^{(\varepsilon)}$, or $c_{\alpha}^{(1)}$ and $c_{\alpha}^{(\varepsilon)}$ are treated as independent ones.

We reconsider the previous example that $\tilde{C}_{1}=\sum_{\alpha=1}^{3} A_{1 \alpha} c_{\alpha}$ and $\tilde{C}_{2}=\sum_{\alpha=1}^{3} A_{2 \alpha} c_{\alpha}$ with $\left|A_{1 \alpha}\right|=O(1),\left|A_{2 \alpha}\right|=O(1)$, and $\left|c_{\alpha}\right|=O(1)$. If $c_{\alpha}$ are given by Eq. (8) and $\sum_{\alpha=1}^{3} A_{2 \alpha} c_{\alpha}^{(1)}=0$ holds, we obtain the hierarchy in magnitude such that

$$
\left|\tilde{C}_{1}\right|=\left|\sum_{\alpha=1}^{3}\left(A_{1 \alpha} c_{\alpha}^{(1)}(f)+A_{1 \alpha} c_{\alpha}^{(\varepsilon)}(f, \varepsilon)\right)\right|=O(1), \quad\left|\tilde{C}_{2}\right|=\left|\sum_{\alpha=1}^{3} A_{2 \alpha} c_{\alpha}^{(\varepsilon)}(f, \varepsilon)\right|=O(\varepsilon) .
$$

The absence of $c_{\alpha}^{(1)}$ in $\tilde{C}_{2}$ could be related to some symmetries.

In this way, we arrive at the idea that parameters in an effective QFT are not necessarily fundamental but effective ones, and then a tininess of some physical parameters can be naturally understood by using more fundamental parameters.

Strictly speaking, we decipher a mechanism that a hierarchy among physical parameters can be realized at a tree level without severe fine tuning. Physical parameters, in general, receive radiative corrections, and hence we need to examine whether the hierarchy is stabilized against radiative corrections or not. Some symmetries can play a central role to the stabilization, and we suppose that they function effectively in our case.

\section{Consideration of fine tuning on Yukawa couplings}

\subsection{Quark Yukawa couplings}

The quark Yukawa coupling matrices $y^{(u)}$ and $y^{(d)}$ are diagonalized bi-unitary transformations as

$$
\begin{aligned}
& V_{\mathrm{L}}^{(u)} y^{(u)} V_{\mathrm{R}}^{(u)^{\dagger}}=y_{\text {diag }}^{(u)}=\operatorname{diag}\left(y_{u}, y_{c}, y_{t}\right), \\
& V_{\mathrm{L}}^{(d)} y^{(d)} V_{\mathrm{R}}^{(d)}{ }^{\dagger}=y_{\text {diag }}^{(d)}=\operatorname{diag}\left(y_{d}, y_{s}, y_{b}\right),
\end{aligned}
$$

where $V_{\mathrm{L}}^{(u)}, V_{\mathrm{L}}^{(d)}, V_{\mathrm{R}}^{(u)}$ and $V_{\mathrm{R}}^{(d)}$ are unitary matrices, and $y_{u}, y_{c}, y_{t}, y_{d}, y_{s}$ and $y_{b}$ are Yukawa couplings of up, charm, top, down, strange, and bottom quarks, respectively. The Kobayashi-Maskawa (KM) matrix is defined by [25]

$$
V_{\mathrm{KM}} \equiv V_{\mathrm{L}}^{(u)} V_{\mathrm{L}}^{(d)^{\dagger}} \text {. }
$$

Each quark mass is obtained by multiplying each Yukawa coupling by the VEV of neutral component of Higgs doublet. From Eqs. (12), (13) and experimental values of quark masses, $y_{\text {diag }}^{(u)}, y_{\text {diag }}^{(d)}$, and $V_{\mathrm{KM}}$ are roughly estimated at the weak scale as [26]

$$
\begin{aligned}
& y_{\text {diag }}^{(u)}=\operatorname{diag}\left(1.3 \times 10^{-5}, 7.3 \times 10^{-3}, 1.0\right)=\operatorname{diag}\left(\lambda^{7}, \lambda^{4}, 1\right), \\
& y_{\text {diag }}^{(d)}=\operatorname{diag}\left(2.7 \times 10^{-5}, 5.5 \times 10^{-4}, 2.4 \times 10^{-2}\right)=\operatorname{diag}\left(\lambda^{7}, \lambda^{5}, \lambda^{3}\right), \\
& V_{\mathrm{KM}}=\left(\begin{array}{ccc}
1 & \lambda & \lambda^{4} \\
\lambda & 1 & \lambda^{2} \\
\lambda^{3} & \lambda^{2} & 1
\end{array}\right),
\end{aligned}
$$


where $\lambda^{n}$ means $O\left(\lambda^{n}\right)$ with $\lambda=\sin \theta_{\mathrm{C}} \cong 0.225$ ( $\theta_{\mathrm{C}}$ is the Cabibbo angle [27]).

Information on physics beyond the SM is hidden in $V_{\mathrm{L}}^{(u)}, V_{\mathrm{R}}^{(u)}$, and $V_{\mathrm{R}}^{(d)}$ besides observable parameters $y_{\text {diag, }}^{(u)}, y_{\text {diag }}^{(d)}$, and $V_{\mathrm{KM}}$. The matrices $V_{\mathrm{L}}^{(u)}, V_{\mathrm{R}}^{(u)}$, and $V_{\mathrm{R}}^{(d)}$ are completely unknown in the SM, because they can be eliminated by the global $U(3) \times U(3) \times$ $\mathrm{U}(3) / \mathrm{U}(1)$ symmetry that the quark kinetic term possesses.

Using $V_{\mathrm{L}}^{(u)}$ and $V_{\mathrm{L}}^{(d)}$, the Hermitian matrices $y^{(u)} y^{(u)^{\dagger}}$ and $y^{(d)} y^{(d)^{\dagger}}$ are diagonalized by unitary transformations:

$$
V_{\mathrm{L}}^{(u)}\left(y^{(u)} y^{(u)^{\dagger}}\right) V_{\mathrm{L}}^{(u)^{\dagger}}=\left(y_{\text {diag }}^{(u)}\right)^{2}, V_{\mathrm{L}}^{(d)}\left(y^{(d)} y^{(d)^{\dagger}}\right) V_{\mathrm{L}}^{(d)^{\dagger}}=\left(y_{\text {diag }}^{(d)}\right)^{2} .
$$

If Yukawa coupling matrices are specified, we can obtain $V_{\mathrm{L}}^{(u)}$ and $V_{\mathrm{L}}^{(d)}$ from (18) and check whether they provide correct KM matrices or not.

As seen from (12), $y_{u}, y_{c}$, and $y_{t}$ are written as linear combinations of $y^{(u)}$ :

$$
y_{u}=\sum_{i, j} R_{11}{ }^{i j} y_{i j}^{(u)}, y_{c}=\sum_{i, j} R_{22}{ }^{i j} y_{i j}^{(u)}, y_{t}=\sum_{i, j} R_{33}{ }^{i j} y_{i j}^{(u)},
$$

where $i, j(=1,2,3)$ are family labels and $R_{i^{\prime} j^{\prime}}{ }^{i j}=\left(V_{\mathrm{L}}^{(u)}\right)_{i^{\prime} i}\left(V_{\mathrm{R}}^{(u)}\right)_{j j^{\prime}}$. When we regard $y_{i j}^{(u)}$ as fundamental parameters, a large cancellation seems likely necessary to obtain $y_{u}=$ $O\left(10^{-5}\right)$ and $y_{c}=O\left(10^{-2}\right)$ in the case with $\left|R_{11}{ }^{i j}\right|=O(1),\left|R_{22}{ }^{i j}\right|=O(1)$, and $\left|y_{i j}^{(u)}\right|=O(1)$ for their non-vanishing components.

\subsection{Reexamination of Yukawa coupling hierarchy}

Let us reexamine the hierarchy among up-type quark Yukawa couplings, based on a general argument in Sect. 2. We consider a simple case with three fundamental parameters $y, \varepsilon_{1}$, and $\varepsilon_{2}$, and asuume that $y_{i j}^{(u)}$ are composed of three parts with much different magnitudes:

$$
y_{i j}^{(u)}=y_{i j}^{u(1)}(y)+y_{i j}^{u\left(\varepsilon_{1}\right)}\left(y, \varepsilon_{1}\right)+y_{i j}^{u\left(\varepsilon_{2}\right)}\left(y, \varepsilon_{1}, \varepsilon_{2}\right),
$$

where $\varepsilon_{1}$ and $\varepsilon_{2}$ are tiny parameters, i.e., $y(=O(1)) \gg \varepsilon_{1}\left(=O\left(\lambda^{4}\right)\right) \gg \varepsilon_{2}\left(=O\left(\lambda^{7}\right)\right)$.

In this case, the hierarchy can be generated without severe fine tuning, in the following setting (a) - (c).

(a) The magnitude of non-vanishing components in $y_{i j}^{u(1)}(y)$ is at most $O(1)$, the rank of $y_{i j}^{u(1)}(y)$ is one, and the magnitude of non-zero eigenvalue is $O(1)$.

(b) The magnitude of non-vanishing components in $y_{i j}^{u\left(\varepsilon_{1}\right)}\left(y, \varepsilon_{1}\right)$ is at most $O\left(\varepsilon_{1}\right)$. The rank of $y_{i j}^{u(1)}(y)+y_{i j}^{u\left(\varepsilon_{1}\right)}\left(y, \varepsilon_{1}\right)$ is two, and the magnitude of non-zero eigenvalues are $O(1)$ and $O\left(\varepsilon_{1}\right)$.

(c) The magnitude of non-vanishing components in $y_{i j}^{u\left(\varepsilon_{2}\right)}\left(y, \varepsilon_{1}, \varepsilon_{2}\right)$ is at most $O\left(\varepsilon_{2}\right)$. 
In fact, under reasonable assumptions such that

$$
\begin{aligned}
& \left|\frac{\partial y^{\left.u\left(\varepsilon_{2}\right)\right)}}{\partial y}\right|=O\left(\varepsilon_{2}\right),\left|\frac{\partial y^{\left.u\left(\varepsilon_{2}\right)\right)}}{\partial \varepsilon_{1}}\right|=O\left(\varepsilon_{2}\right), \quad\left|\frac{\partial y^{\left.u\left(\varepsilon_{2}\right)\right)}}{\partial \varepsilon_{2}}\right|=O(1), \\
& \left|\frac{\partial y^{\left.u\left(\varepsilon_{1}\right)\right)}}{\partial y}\right|=O\left(\varepsilon_{1}\right),\left|\frac{\partial y^{\left.u\left(\varepsilon_{1}\right)\right)}}{\partial \varepsilon_{1}}\right|=O(1),
\end{aligned}
$$

degrees of fine tuning for $y_{u}$ and $y_{c}$ are estimated as

$$
\begin{aligned}
& \Delta_{y} y_{u} \equiv\left|\frac{\partial \ln y_{u}}{\partial \ln y}\right|=O(1), \Delta_{\varepsilon_{1}} y_{u} \equiv\left|\frac{\partial \ln y_{u}}{\partial \ln \varepsilon_{1}}\right|=O\left(\varepsilon_{1}\right), \Delta_{\varepsilon_{2}} y_{u} \equiv\left|\frac{\partial \ln y_{u}}{\partial \ln \varepsilon_{2}}\right|=O(1), \\
& \Delta_{y} y_{c} \equiv\left|\frac{\partial \ln y_{c}}{\partial \ln y}\right|=O(1), \Delta_{\varepsilon_{1}} y_{c} \equiv\left|\frac{\partial \ln y_{c}}{\partial \ln \varepsilon_{1}}\right|=O(1), \Delta_{\varepsilon_{2}} y_{c} \equiv\left|\frac{\partial \ln y_{c}}{\partial \ln \varepsilon_{2}}\right|=O\left(\varepsilon_{2} / \varepsilon_{1}\right),
\end{aligned}
$$

and they suggest that the large hierarchy with $y_{t}=O(1), y_{c}=O\left(\varepsilon_{1}\right)$, and $y_{u}=O\left(\varepsilon_{2}\right)$ is naturally realized.

Finally, let us give an illustration with a matrix given by

$$
y_{i j}^{(u)}=y S_{i j}+y_{i j}^{u\left(\varepsilon_{1}\right)}\left(y, \varepsilon_{1}\right)+y_{i j}^{u\left(\varepsilon_{2}\right)}\left(y, \varepsilon_{1}, \varepsilon_{2}\right),
$$

where $S_{i j}$ is the $(i, j)$ component of the democratic matrix defined by

$$
S \equiv \frac{1}{3}\left(\begin{array}{lll}
1 & 1 & 1 \\
1 & 1 & 1 \\
1 & 1 & 1
\end{array}\right) .
$$

$S$ is easily diagonalized as $U S U^{\dagger}=\operatorname{diag}(0,0,1)$ with the unitary matrix:

$$
U=\frac{1}{\sqrt{3}}\left(\begin{array}{ccc}
\bar{\omega} & \omega & 1 \\
\omega & \bar{\omega} & 1 \\
1 & 1 & 1
\end{array}\right),
$$

where $\omega=e^{2 \pi i / 3}$ and $\bar{\omega}=\omega^{2}=e^{4 \pi i / 3} \cdot y_{i j}^{u\left(\varepsilon_{1}\right)}$ and $y_{i j}^{u\left(\varepsilon_{2}\right)}$ are supposed to be given by polynomials of $\varepsilon_{1}$ and $\varepsilon_{2}$ such that

$$
y_{i j}^{u\left(\varepsilon_{1}\right)}\left(y, \varepsilon_{1}\right)=\sum_{n=1} c_{i j, n}^{\left(\varepsilon_{1}\right)}(y) \varepsilon_{1}^{n}, y_{i j}^{u\left(\varepsilon_{2}\right)}\left(y, \varepsilon_{1}, \varepsilon_{2}\right)=\sum_{n=1} c_{i j, n}^{\left(\varepsilon_{2}\right)}\left(y, \varepsilon_{1}\right) \varepsilon_{2}^{n} .
$$

Then, $y_{i j}^{(u)}$ is diagonalized as

$$
V_{\mathrm{L}}^{(u)}\left(y S+y^{u\left(\varepsilon_{1}\right)}\left(y, \varepsilon_{1}\right)+y^{u\left(\varepsilon_{2}\right)}\left(y, \varepsilon_{1}, \varepsilon_{2}\right)\right) V_{\mathrm{R}}^{(u)^{\dagger}}=\operatorname{diag}\left(y_{u}, y_{c}, y_{t}\right),
$$

where $V_{\mathrm{L}}^{(u)}, V_{\mathrm{R}}^{(u)}, y_{u}, y_{c}$, and $y_{t}$ are perturbatively given by

$$
\begin{aligned}
& \left(V_{\mathrm{L}}^{(u)}\right)_{i j}=U_{i j}+\sum_{n=1}^{\infty} c_{i j, n}^{\left(\mathrm{L}, \varepsilon_{1}\right)}(y) \varepsilon_{1}^{n}+\sum_{n=1}^{\infty} c_{i j, n}^{\left(\mathrm{L}, \varepsilon_{2}\right)}\left(y, \varepsilon_{1}\right) \varepsilon_{2}^{n}, \\
& \left(V_{\mathrm{R}}^{(u)}\right)_{i j}=U_{i j}+\sum_{n=1}^{\infty} c_{i j, n}^{\left(\mathrm{R}, \varepsilon_{1}\right)}(y) \varepsilon_{1}^{n}+\sum_{n=1}^{\infty} c_{i j, n}^{\left(\mathrm{R}, \varepsilon_{2}\right)}\left(y, \varepsilon_{1}\right) \varepsilon_{2}^{n},
\end{aligned}
$$




$$
\begin{aligned}
& y_{u}=\sum_{n=1}^{\infty} c_{n}^{\left(u, \varepsilon_{2}\right)}\left(y, \varepsilon_{1}\right) \varepsilon_{2}^{n}, \quad y_{c}=\sum_{n=1}^{\infty} c_{n}^{\left(c, \varepsilon_{1}\right)}(y) \varepsilon_{1}^{n}+\sum_{n=1}^{\infty} c_{n}^{\left(c, \varepsilon_{2}\right)}\left(y, \varepsilon_{1}\right) \varepsilon_{2}^{n}, \\
& y_{t}=y+\sum_{n=1}^{\infty} c_{n}^{\left(t, \varepsilon_{1}\right)}(y) \varepsilon_{1}^{n}+\sum_{n=1}^{\infty} c_{n}^{\left(t, \varepsilon_{2}\right)}\left(y, \varepsilon_{1}\right) \varepsilon_{2}^{n} .
\end{aligned}
$$

Degrees of fine tuning for $y_{u}$ and $y_{c}$ are estimated as

$$
\begin{aligned}
& \Delta_{y} y_{u}=O(1), \Delta_{\varepsilon_{1}} y_{u}=O\left(\varepsilon_{1}\right), \Delta_{\varepsilon_{2}} y_{u}=O(1), \\
& \Delta_{y} y_{c}=O(1), \Delta_{\varepsilon_{1}} y_{c}=O(1), \Delta_{\varepsilon_{2}} y_{c}=O\left(\varepsilon_{2} / \varepsilon_{1}\right),
\end{aligned}
$$

using $\left|c_{1}^{\left(u, \varepsilon_{2}\right)}\right|=O(1),\left|\partial c_{1}^{\left(u, \varepsilon_{2}\right)} / \partial y\right|=O(1),\left|\partial c_{1}^{\left(u, \varepsilon_{2}\right)} / \partial \varepsilon_{1}\right|=O(1),\left|c_{1}^{\left(c, \varepsilon_{1}\right)}\right|=O(1),\left|c_{1}^{\left(c, \varepsilon_{2}\right)}\right|=$ $O(1),\left|\partial c_{1}^{\left(c, \varepsilon_{1}\right)} / \partial y\right|=O(1),\left|\partial c_{1}^{\left(c, \varepsilon_{2}\right)} / \partial y\right|=O(1)$, and $\left|\partial c_{1}^{\left(c, \varepsilon_{2}\right)} / \partial \varepsilon_{1}\right|=O(1)$. From Eqs. (34) and (35), there seems no severe fine tuning to derive $y_{u}$ and $y_{c}$. Note that no contributions of $y_{i j}^{u(1)}(y)=y S_{i j}$ in $y_{u}$ and $y_{c}$ stem from $\left(U S U^{\dagger}\right)_{11}=0$ and $\left(U S U^{\dagger}\right)_{22}=0$ relating to $S_{3}$ symmetry.

\section{Conclusions and discussions}

We have reconsidered a generation of up-type quark mass hierarchy in the SM, and clarified how a mechanism works that the hierarchy is realized without severe fine tuning. Based on the idea that parameters in an effective QFT are not necessarily fundamental but effective ones, and a tininess of physical parameters can be naturally understood by using more fundamental parameters, we have found that up-type quark mass hierarchy can be naturally realized, if up-type Yukawa coupling matrix consists of several parts with much different magnitudes, the rank of a dominant part is one, and the rank of a sum of dominant and semi-dominant ones is two.

The mechanism is available for the generation of both up-type and down-type quark mass hierarchies in an extension of the SM with extra vector-like fermions. We consider a case with $n$ families of quarks and $n-3$ families of mirror quarks. It is assumed that $n \times n$ up-type Yukawa coupling matrix consists of $y^{u(1)}(=O(1))$ and other tiny ones, and $n \times n$ down-type Yukawa coupling matrix also consists of $y^{d(1)}(=O(1))$ and other tiny ones. If the rank of the dominant part $y^{u(1)}$ is $n-2$ and that of $y^{d(1)}$ is $n-3$, there can appear two up-type quarks and three down-type quarks much below the weak scale. The $n-3$ sets of up-type and down-type quarks form vector-like heavy fermions in company with mirror ones. Then an up-type quark with a Yukawa coupling of $O(1)$ remains as a chiral one, it acquires a mass of the weak scale after the breakdown of electroweak symmetry, and it is identified as a top quark.

It would be interesting to study a mechanism behind the flavor structure, from the aspect of an exploration of a theory beyond the SM. As a by-product, we might close in on an unknown part of QFTs through generic features in the mechanism.

\section{Acknowledgments}

This work was supported in part by scientific grants from the Ministry of Education, Culture, Sports, Science and Technology under Grant No. 17K05413. 


\section{References}

[1] M.S. Chanowitz, J. Ellis, and M.K. Gailard, Nucl. Phys. B 129, 506 (1977).

[2] H. Fritzsch, Phys. Lett. B 73, 317 (1978).

[3] H. Harari, H. Haut, and J. Weyers, Phys. Lett. B 78, 459 (1978).

[4] H. Fritzsch, Nucl. Phys. B 155, 189 (1979).

[5] H. Georgi and C. Jarlskog, Phys. Lett. B 86, 297 (1979).

[6] C.D. Froggatt and H.B. Nielsen, Nucl. Phys. B 147, 277 (1979).

[7] T. Maehara and T. Yanagida, Prog. Theor. Phys. 60, 822 (1978).

[8] K. Inoue, Prog. Theor. Phys. 93, 403 (1995).

[9] P.F. Harrison, D.H. Perkins, and W.G. Scott, Phys. Lett. B 530, 167 (2002).

[10] P.F. Harrison and W.G. Scott, Phys. Lett. B 535, 163 (2002).

[11] M. Kakizaki and M. Yamaguchi, Phys. Lett. B 573, 123 (2003).

[12] G. Altareli and F. Feruglio, Rev. Mod. Phys. 82, 2701 (2010).

[13] H. Ishimori, T. Kobayashi, H. Ohki, Y. Shimizu, H. Okada, and M. Tanimoto, Prog. Theor. Phys. Suppl. 183, 1 (2010).

[14] H. Ishimori, T. Kobayashi, H. Ohki, H. Okada, Y. Shimizu, and M. Tanimoto, Lect. Note. Phys. 858, 1 (2012) and references therein.

[15] M. Leurer, Y. Nir, and N. Seiberg, Nucl. Phys. B 398, 319 (1993).

[16] Y. Koide, Phys. Rev. D 71, 016010 (2005).

[17] A. Kusenko, Phys. Lett. B 284, 390 (1992).

[18] C.H. Albright and S. Nandi, Phys. Rev. Lett. 73, 930 (1994).

[19] D. Marzocca and A. Romanino, J. High Energy Phys. 11, 159 (2014).

[20] V. Domeke and A. Romanino, J. High Energy Phys. 06, 031 (2016).

[21] Y. Kawamura, Prog. Theor. Exp. Phys. 2019, $043 B 05$ (2019).

[22] Y. Kawamura, Prog. Theor. Exp. Phys. 2019, 073B03 (2019).

[23] Y. Kawamura, to appear in Prog. Theor. Exp. Phys. 2020 [arXiv:1909.03623[hep-ph]].

[24] R. Barbieri and G. F. Giudice, Nucl. Phys. B 306, 63 (1988).

[25] M. Kobayashi and T. Maskawa, Prog. Theor. Phys. 49, 652 (1973). 
[26] C. Patrignani et al. [Particle Date Group], Chin. Phys. C 40, 100001 (2016).

[27] N. Cabibbo, Phys. Rev. Lett. 10, 531 (1963). 